\title{
Minimally Invasive Techniques for Treating Gynecologic Malignancies
}

\author{
Presented by Amanda N. Fader, MD
}

\section{Abstract}

For many women with endometrial and cervical cancers, minimally invasive surgery represents an alternative standard of care to open abdominal procedures, with fewer complications, better postoperative quality of life, and overall lower cost. At the NCCN 22nd Annual Conference, Amanda N. Fader, MD, reviewed several minimally invasive strategies for treating gynecologic cancers, including conventional laparoscopy, robotic-assisted laparoscopy, sentinel lymph node technology, and single-port surgery; highlighted some of the contemporary literature on the role of these procedures; and explored some of the challenges and barriers to their successful performance.

J Natl Compr Canc Netw 2017;15(5.5):730-732

"We can offer less invasive procedures to our female patients with below-the-belt cancers without compromising oncologic outcomes," announced Amanda N. Fader, MD, Director, Kelly Gynecologic Oncology Service, and Associate Professor, Gynecology and Obstetrics, The Johns Hopkins School of Medicine. In fact, less may be more when it comes to minimally invasive surgery (MIS) for the primary treatment of certain gynecologic cancers, she added. Dr. Fader is an editorial board member for Gynecologic Oncology and serves on the NCCN Cervical/Uterine/Vulvar Cancers Panel.

Although MIS was initially used solely to aid in establishing a cancer diagnosis, it has now graduated to therapeutics, with various roles including staging, defining the extent of adjuvant therapy, and managing locoregional recurrence. In fact, laparoscopy has become the gold standard treatment of many gynecologic conditions, "both benign and malignant," reported Dr. Fader. Many studies have shown improved outcomes with MIS, ${ }^{1,2}$ and a host of benefits have been reported, including fewer perioperative and postoperative complications, shorter hospital stays, improved cosmesis, faster recovery, and decreased costs. Furthermore, MIS may be beneficial for obese or elderly patients with many

Presented by Amanda N. Fader, MD, Kelly Gynecologic Oncology Service, The Johns Hopkins School of Medicine, Baltimore, Maryland. Dr. Fader has disclosed that she has received consulting fees or honoraria from Merck \& Co., Inc., and served as a scientific advisor for Genentech, Inc.

Correspondence: Amanda N. Fader, MD, 600 North Wolfe Street, Phipps 287, Baltimore, MD 21287. E-mail: afader1@jhmi.edu comorbidities, and less physical and psychologic trauma is another advantage.

However, admitted Dr. Fader, minimally invasive procedures have not been completely generalizable. "Despite level 1 evidence showing the benefits of laparoscopic hysterectomy and other minimally invasive procedures, there is still a 50\% abdominal hysterectomy rate," she said.

\section{Clinical Indications}

Dr. Fader detailed the role of conventional laparoscopy in the treatment of endometrial, cervical, and ovarian cancers and then reviewed recommendations for its use in certain clinical scenarios.

\section{Endometrial Cancer}

The most common gynecologic malignancy is endometrial cancer; approximately $75 \%$ of these cancers are early-stage tumors. Many of these cancers can be cured with surgery alone; thus, total abdominal hysterectomy/ bilateral salpingo-oophorectomy and sometimes pelvic and para-aortic lymphadenectomy represent the historical standard of care. ${ }^{2}$ However, a new standard of care has emerged for early-stage endometrial carcinoma, and the 2017 NCCN Clinical Practice Guidelines in Oncology (NCCN Guidelines) for Uterine Neoplasms note that "MIS is the preferred approach when technically feasible." ${ }^{3}$ This recommendation is based on 8 randomized controlled trials and 2 meta-analyses, with level 1 evidence $^{4}$ demonstrating no difference in survival, Dr. Fader added. 
Gynecologic Malignancies

Dr. Fader noted that at The Sidney Kimmel Comprehensive Cancer Center at Johns Hopkins, MIS was used in $>90 \%$ of endometrial cases from 2012 to 2016. In addition, MIS was used in $>80 \%$ of obese women. "Endometrial cancer is the most obesitydriven malignancy in men or women," she noted, and $65 \%$ have obesity-driven comorbidities.

Regarding high-grade endometrial cancer, Dr. Fader noted that this type of tumor is potentially more comorbid, with a higher risk of lymph node involvement, systemic involvement, and recurrence. According to her center's data, ${ }^{5}$ women with high-grade endometrial cancers staged using MIS experienced fewer complications and similar survival outcomes compared with those staged using laparotomy.

\section{Cervical Cancer}

Although cervical cancer is less common than endometrial cancer, it still represents a significant clinical problem in the United States. Approximately half of these tumors are diagnosed at an early stage, and in such cases, "we can offer less radical hysterectomies," Dr. Fader said. At Johns Hopkins, from 2012 to 2016 , MIS was performed in nearly $80 \%$ of cervical cancer cases. However, women who have tumors from 1 to $4 \mathrm{~cm}$ are offered radical hysterectomy. "We do not have phase III data available to support MIS in this population," she noted.

\section{Ovarian Cancer}

Unlike the other 2 gynecologic malignancies discussed, ovarian cancer is often diagnosed at an advanced stage, so the gold standard of treatment remains primary cytoreductive surgery followed by platinum-taxane chemotherapy, according to the NCCN Guidelines for Ovarian Cancer. Although Dr. Fader noted that at Johns Hopkins from 2012 to 2016 MIS was used in $<20 \%$ of ovarian cancer cases, she said that conventional laparoscopic surgical staging is feasible and safe, and helps determine whether a patient is a candidate for primary cytoreductive surgery. ${ }^{6}$ "For staging or restaging, laparoscopy is a nice alternative," she indicated.

\section{Robotic-Assisted Laparoscopy}

Dr. Fader acknowledged that laparoscopy is associated with some problems: the screens are 2dimensional, the scope is unstable, the instrumentation is long and rigid, the technique can be ergonomically challenging for surgeons, and techniques have remained static in the past decade. The use of robotics in the laparoscopic procedure addresses some of these issues. For instance, it offers 3-dimensional, high-definition vision with zoom capabilities and a stable scope; articulated instrumentation with 7 degrees of freedom; and optimized ergonomics.

Robotic-assisted laparoscopy is an effective surgical alternative for obese patients with endometrial cancer, associated with significantly shorter surgical times, lower estimated blood loss, increased lymph node count, and shorter hospital stay according to clinical trials in the literature.? "We try to use laparoscopic procedures for straightforward cases and for thinner women," she explained. "For severely obese women, who may have difficult anatomy or adhesions, a good argument can be made in terms of health care utilization of resources to use robotic surgery."

However, a key component to successful robotic surgery is a well-trained surgeon with extensive experience to perform these procedures. Consequently, training opportunities for residents and fellows are essential. Finally, although rare, mechanical failures can occur, she noted, "so surgeons need to be savvy in both laparoscopic techniques and robotic techniques."

\section{Other Surgical Alternatives}

Sentinel lymph node (SLN) mapping technology and single-port surgery are other surgical alternatives for assessing or treating gynecologic malignancies. These techniques are evolving and warrant further exploration.

According to Dr. Fader, the rationale behind using SLN mapping in this patient population centers mainly on several factors: node status is the most important predictor of survival in vulvar, cervical, and endometrial cancers; $75 \%$ of patients with early-stage tumors are node-negative and would benefit from avoiding complete lymphadenectomy; and there is a reduced risk of lymphedema and vascular injuries.

As a result, the NCCN Guidelines for Uterine Neoplasms (which include discussion of endometrial cancer) included the following statement regarding SLN mapping as an alternative standard of care: "SLN mapping can be considered for the surgical staging of 
Fader

apparent uterine-confined malignancy when there is no metastasis demonstrated by imaging studies or no obvious extrauterine disease at exploration."

Dr. Fader briefly reviewed the surgical technique for identifying the SLN through the injection of isosulfan blue or indocyanine green dyes. ${ }^{8}$ "You can see these gorgeous channels coursing through the retroperitoneum to identify the lymph node," she noted. However, she added, SLN mapping appears to be better with the green dye, particularly in obese patients. "The green dye is easier to see and overcomes some anatomic limitations," she said.

Based on level 2 to 3 evidence, "the NCCN has now adopted this [SLN biopsy] based on data from Memorial Sloan Kettering Cancer Center, Hopkins, University of North Carolina, Canada, and France about this being an acceptable alternative standard of care in smaller tumors," Dr. Fader said. However, she cautioned that this technique is not without its limitations, such as requiring surgical experience to attain competency and the fact that gynecologic organs have a complex lymphatic drainage system.

Finally, single-port surgery represents a promising possible future alternative. Laparoendoscopic singlesite surgery is an even less invasive option to conventional or robotic laparoscopy. With the surgeon operating almost exclusively through a single, small entry at the patient's navel, the morbidity associated with multiple incisions, such as pain, infection, hernia, and scarring, is reduced. Preliminary outcomes with this approach in gynecologic, urologic, and general surgery are encouraging, suggested Dr. Fader. "[Single-port surgery] can be used to remove cysts or masses," she added. "It produces minimal scarring."

\section{References}

1. Johnson N, Barlow D, Lethaby A, et al. Surgical approach to hysterectomy for benign gynaecological disease. Cochrane Database Syst Rev 2006:CD003677.

2. Scalici J, Laughlin BB, Finan MA, et al. The trend towards minimally invasive surgery (MIS) for endometrial cancer: an ACS-NSQIP evaluation of surgical outcomes. Gynecol Oncol 2015;136:512-515.

3. Koh WJ, Greer B, Abu-Rustum NR, et al. NCCN Clinical Practice Guidelines in Oncology: Uterine Neoplasms, Version 1.2017. Accessed April 14, 2017. To view the most recent version of these guidelines, visit NCCN.org.

4. Palomba S, Falbo A, Mocciaro R, et al. Laparoscopic treatment for endometrial cancer: a meta-analysis of randomized controlled trials. Gynecol Oncol 2009;112:415-421.
5. Fader AN, Seamon LG, Escobar PF, et al. Minimally invasive surgery versus laparotomy in women with high grade endometrial cancer: a multi-site study performed at high volume cancer centers. Gynecol Oncol 2012;126:180-185.

6. Walker JL, Piedmonte MR, Spirtos NM, et al. Laparoscopy compared with laparotomy for comprehensive surgical staging of uterine cancer: Gynecologic Oncology Group Study LAP2. J Clin Oncol 2009;27:53315336.

7. Gala RB, Margulies R, Steinberg A, et al. Systematic review of robotic surgery in gynecology: robotic techniques compared with laparoscopy and laparotomy. J Minim Invasive Gynecol 2014;21:353-361.

8. Beavis AL, Salazar-Marioni S, Sinno AK, et al. Sentinel lymph node detection rates using indocyanine green in women with early-stage cervical cancer. Gynecol Oncol 2016;143:302-306. 